greater role in policymaking across a range of sectors. We have seen the positive results of applying this scientific knowledge in diverse areas of public policy such as taxation, energy, and education, among others. The insights used to design these interventions were evidence-based cognitive and psychosocial discoveries such as the existence of cognitive biases and the importance of social norms in human behavior.

Behavioral units needed to show significant results for a minimum or zero cost in order to prove their worth and thus, cement the incorporation of behavioral sciences into the public sphere. It is time to move on to a renewed level of complexity by designing more comprehensive interventions with deeper implications and long-term results.

The role of neurosciences has been much less explored but shows a promising potential to improve public policy, especially social policy. For instance, the design of anti-poverty programs based on scientific evidence about brain health would be extremely useful to improve the mental capital of persons living in poverty. Findings in neurosciences along the life cycle can be incorporated into the public policy sphere. Understanding the neuroscientific evidence on how to stimulate cognitive and socio-emotional skills among different types of populations is crucial to improve the design of policies and has relevant implications for the mental capital of nations.

Neurosciences can also facilitate the comprehension of those cognitive skills needed for the jobs of the future such as creativity, emotional intelligence, empathy, resilience, cognitive flexibility, and executive functions, among others. Public policy interventions can focus on promoting these skills, which are not fixed and which can be improved and enhanced. Other areas in which evidence from neurosciences can be utilized are: infant development, the adolescent brain, addictions (i.e., opioid epidemic, science and policy of marijuana), neuroeducation or the neuroscientific basis in teaching and learning (i.e., dyslexia), neurolaw (i.e. criminal responsibility, deception, juvenile justice, judges' decision making, witness testimony), prejudice and empathy, violence and aggression, loneliness, normal aging, the future of mind-tech cyborgs, and the burden of brain disorders.

Behavioral insights are gaining popularity in developed countries. However, is this enough to create positive structural changes in the economy and in social welfare? While it is fair to say that nudges successfully allowed behavioral science to enter the world of policy we still have much work to do to reach social and health long-term improvements. In this regard, advances in neurosciences over the last years could help reshape the way we think about important policies and could be useful in helping individuals develop their maximum potential.

\section{CULTURE ETHNIC MINORITIES AND HEALTH}

CBE Dinesh Bhugra. Emeritus Professor of Mental Health and Cultural Diversity at the Institute of Psychiatry, Psychology and Neuroscience at King's College London

\subsection{6/jnnp-2019-BNPA.8}

Professor Dinesh Bhugra was President of the World Psychiatric Association from 2014-2017 and in 2018 became President of the British Medical Association. Dinesh Bhugra's research interests are in cultural psychiatry, sexual dysfunction and service development. He is the recipient of over 10 honorary degrees. He has authored/co-authored over 400 scientific papers and 30 books, and is the Editor of three journals (International Journal of Social Psychiatry, International Review of Psychiatry and International Journal of Culture and Mental Health). Previously he was the Dean (2003-2008) and then President (2008-2011) of the Royal College of Psychiatrists in the UK, where he led on major policy initiatives on psychiatry's contract with society and the role of the psychiatrist.

Culture form us and we form culture. Culture influences the way we think and the way we see the world. Furthermore, recognition and presentation of distress as well as patterns of help seeking are very strongly influenced by cultures and our world views. The accessibility and availability of healthcare system also influences when and where we seek help from. Cultures include religion, spirituality, language, diet, dress etc. In many cultures there are no words to express many psychiatric and neurological disorders even though symptoms of distress are easily recognised. In addition, depending upon the explanatory models of distress patients and their families may seek help from non-professional sources. Studies from the USA have suggested that a vast majority of people seek help from personal, folk or social sectors and only a minority reach the professional health sector. This may reflect the American healthcare system. Gender and other factors such as education, social class, housing, employment and economic factors contribute to help-seeking. Cultural identity and embedded within are micro-identities which help formulate an unique cultural framework which differentiates one group from another and can work at both individual and group levels. Using various models this lecture will explore varying presentations and using examples from around the world will illustrate cultural impact on various neurological and psychiatric disorders. Community support in various forms can enable quick recovery and help support vulnerable groups.

\section{POVERTY AND BRAIN DEVELOPMENT: FROM SCIENCE TO POLICY}

${ }^{1}$ Martha J Farah, ${ }^{2}$ Walter H Annenberg. 'Director, Center for Neuroscience and Society; ${ }^{2}$ Professor in the Natural Sciences, University of Pennsylvania

\subsection{6/jnnp-2019-BNPA.9}

Martha Farah was born in New York City and educated at MIT and Harvard. She has taught at Carnegie-Mellon University and the University of Pennsylvania, where she is currently the Walter H. Annenberg Professor of Natural Sciences. In 1999 she founded Penn's Center for Cognitive Neuroscience and ten years later she founded the Center for Neuroscience and Society, which she still directs. Martha's work on the ethical, legal and social impact of neuroscience (aka neuroethics) has focused on cognitive enhancement by normal individuals, including the question of whether drugs and devices believed to enhance normal cognition actually do so, and on nonclinical uses of brain imaging. Her other main interest is in the effects of early socioeconomic deprivation on brain development. She studies the latter using behavioral, neuroendocrine and neuroimaging methods. Martha is a Fellow of the American Academy of Arts and Sciences, a former Guggenheim Fellow and recipient of honors including the National Academy of Science's Troland Research Award and the Association for Psychological Science's lifetime achievement award. 
Childhood socioeconomic status (SES) predicts many important life outcomes, from physical and mental health to academic achievement and cognitive ability. Why is SES so influential? Part of the answer lies in the relationship between SES and the brain. I will present research from my lab and others aimed at characterizing SES differences in brain structure and function. I will then discuss the causes of these associations - by what mechanisms are they linked? - and their consequences - what roles do they play in the health and achievement disparities mentioned earlier? Finally, I will consider whether and how the neuroscience of SES can help shape policies concerning children of low SES.

\section{INFLAMED DEPRESSION}

Edward Bullmore. Department of Psychiatry, University of Cambridge RandD, GlaxSmitKline

\subsection{6/jnnp-2019-BNPA.10}

Edward Bullmore, FRCP, FRCPsych, FMedSci began his medical career as an academic rather than a physician. From 1987 to 1988 , he was a lecturer in medicine at the University of Hong Kong. He then returned to England where he began training in his chosen specialisation as a Senior House Officer in psychiatry at St George's Hospital, London. After a year, he moved hospitals, and was appointed a Registrar in psychiatry at Bethlem Royal Hospital and Maudsley Hospital; both specialist psychiatric hospitals in London. In 1993, Bullmore began his research career. That year, he was appointed a Wellcome Trust Research Training Fellow and served in that role for three years. During that time he studied for a Doctor of Philosophy (PhD) degree at King's College London, which he completed in 1997 with a thesis titled 'Analysis of structural and functional magnetic resonance images of the brain'. In 1996, he was promoted to an Advanced Research Training Fellow for a further three years. His research during this time focused on the mathematical analysis of neurophysiological time series. From 1996 to 1999, he was additionally an honorary Consultant Psychiatrist at Maudsley Hospital, London. In 1999, Bullmore joined the University of Cambridge as Professor of Psychiatry. At college level, he was an elected Fellow of Wolfson College, Cambridge between 2002 and 2010. On 9 October 2014, he was appointed Head of the Department of Psychiatry, University of Cambridge. In 2005, he joined GlaxoSmithKline as Vice-President of Experimental Medicine. From 2005 to 2013, he was also Head of its Clinical Unit based in Addenbrooke's Hospital, Cambridge, Cambridgeshire, which focuses on early clinical drug development projects.

It is beyond doubt that inflammation and depression are associated with each other. Many patients with arthritis, or other inflammatory disorders of the body, have increased risk of depression; many patients with depression have increased blood levels of inflammatory proteins like cytokines. I will discuss how this association has traditionally been interpreted (or discounted) in the Cartesian framework of medical science; and I will highlight new evidence for a direct mechanistic relationship, whereby inflammation of the body or brain can cause depressive behaviours. This raises a number of further questions. First, can known risk factors for depression - like social stress - cause inflammation? Second, what is the current evidence that anti-inflammatory interventions can have antidepressant effects and how could new, more personalized approaches to treatment of 'inflamed depression' (not all depression) be developed in future?.

\section{REFERENCE}

Bullmore ET (2018) 'The Inflamed Mind' Short Books, London.

\section{Day $2-8^{\text {th }}$ March 2019}

\section{Functional neurological disorder and it's boundaries, members' platform, BNPA medal lecture}

\section{THE BLADDER AND THE BRAIN: EXPLORING FUNCTIONAL UROLOGICAL SYMPTOMS}

Ingrid Hoeritzauer. Clinical Research Fellow, University of Edinburgh

10.1136/jnnp-2019-BNPA.11

Ingrid Hoeritzauer is a neurologist who also has a degree in psychology. She works with the Functional Research Team led by Jon Stone and Alan Carson in Edinburgh and received an ABN/Patrick Berthoud Clinical Research Training Fellowship. Her research interests are observational studies in functional neurological disorders and cauda equina syndrome and her current research focuses on the interface between Uro-Neurology and functional neurological disorders.

This talk will review the current understanding of the bladder-brain axis, and the evidence linking functional disorders, including Functional Neurological Disorder (FND), to bladder symptoms including urinary retention and in the study of patients who present with cauda equina syndrome but have normal scans. Uro-Neurology is the connection between urological symptoms and the neurological system, comprising a complex bladder-brain network involving the brain, spinal cord, sacral nerves and peripheral (pelvic) nerves. Since the time of Charcot there have been hypotheses that urological symptoms, particularly idiopathic urinary retention and overactive bladder symptoms, were part of a functional disorder. However, in the 1980s urethral EMG changes in women with idiopathic urinary retention led to a view that so called 'psychogenic' urinary retention was a primary disorder of the urethral sphincter. Changing views of FND in the last 10 years mean that urological symptoms are once again being investigated as a potential part of functional disorders. Patients presenting with chronic idiopathic urinary retention, diagnosed as Fowler's syndrome, or presenting with acute urinary retention and possible cauda equina syndrome, have been found to have high levels of functional disorder and FND comorbidity ${ }^{1}{ }^{2}$. Additionally, recent papers have suggested central sensitisation as the mechanism of action in patients with idiopathic overactive bladder symptoms. ${ }^{3}$ This newly re-energised field of study requires further exploration, such as investigation of the effect of pathomechanical and medication induced urological symptoms on patients with functional disorders. A practical guide to how to discuss likely functional urological symptoms based on the current level of knowledge will conclude the talk.

\section{REFERENCES}

1. Hoeritzauer I, Stone J, Fowler C, Elneil-Coker S, Carson A, Panicker J. Fowler's syndrome of urinary retention: A retrospective study of co-morbidity. Neurourol Urodyn 2016;35:601-3. 